\title{
The combined effect of sleep and time of day on emotion decoding from dynamic visual cues in older adults
}

This article was published in the following Dove Press journal:

Neuropsychiatric Disease and Treatment

I September 2016

Number of times this article has been viewed

\author{
Paraskevi Tsokanaki' \\ Despina Moraitou' \\ Georgia Papantoniou ${ }^{2}$ \\ 'Section of Cognitive and \\ Experimental Psychology, School \\ of Psychology, Aristotle University \\ of Thessaloniki, Thessaloniki, \\ ${ }^{2}$ Department of Early Childhood \\ Education, University of Ioannina, \\ loannina, Greece
}

\begin{abstract}
It is well known that night sleep is a decisive factor for the effective functioning of the human body and mind. In addition to the role of sleep, older adults report that they are "morning types" and that their cognitive and emotional abilities seem to be at a higher level in the morning hours. In this vein, this study is aimed at examining the effect of sleep combined with the "time of day" condition on a specific ability that is crucial for interpersonal communication, namely, emotion recognition, in older adults. Specifically, the study compared older adults' performance in decoding emotions from ecologically valid, dynamic visual cues, in two conditions: "early in the morning and after night sleep", and "in the afternoon and after many hours since night sleep". An emotion recognition task was administered twice to 37 communitydwelling older adults. The results showed a statistically significant higher performance in the morning in decoding all emotions presented, compared to the afternoon condition. Pleasant surprise, sadness, and anxiety were revealed as the most difficult emotions to be recognized in the afternoon condition.
\end{abstract}

Keywords: aging, cognition, emotion recognition

\section{Introduction}

\section{Emotion recognition in older adults}

Two basic skills are required in order to achieve an effective social interaction: 1) the expression of our own emotions; and 2) the recognition of others' emotions, mainly the "basic" ones. Certain emotions universally recognized are considered basic emotions. Specifically, the following six emotions are classified as basic: anger, disgust, fear, happiness, sadness, and surprise. ${ }^{1}$ According to the extant literature, emotion recognition - even if it is a relatively primitive process $^{2}$ - requires a series of cognitive processes such as perception, pattern recognition, and long-term memory. All these processes are regulated by the executive functions. Executive functions are primarily supported by the activation of the frontal lobe. Frontal lobe monitors and interacts with other brain structures involved in emotion recognition, such as the temporal areas of the amygdala and the fusiform cortex (which appear to have a general role in responding to facial expressions), the dorsolateral prefrontal cortex, the ventral striatum, and the superior temporal sulcus, as well as the visual processing areas in the parietal and occipital lobes. ${ }^{2-4}$ Emotion recognition is an important skill that substantially supports social cognition and enables the person to understand and predict others' behavior, provide support, and avoid conflict. ${ }^{5}$ Hence, difficulties in emotion recognition are considered a main factor for inadequate communication. Numerous
Correspondence: Despina Moraitou Section of Cognitive and Experimental Psychology, School of Psychology, Aristotle University of Thessaloniki, Thessaloniki 54I 24, Greece $\mathrm{Tel} / \mathrm{fax}+3023$ I 020 I 372 Email demorait@psy.auth.gr 
studies have found that older adults are less able to identify emotions mainly expressed via visual cues, compared to younger adults. ${ }^{6-8}$ Nevertheless, to our knowledge, there are no studies examining the possible effects of sleep and "time of day" (ToD) on older adults' ability to decode the six basic emotions from visual cues, especially, when these cues are presented dynamically, via an ecologically valid way.

\section{The effects of sleep and sleep deprivation on cognition and affect}

The influence of sleep and sleep deprivation on the physiology of human body, especially on brain structures that are involved in cognitive and affective functions, has attracted the interest of researchers in the past decades. Recent research has resulted in important findings regarding the positive effects of sleep, particularly those of the rapid eye movement (REM) stage of sleep, on emotion regulation. ${ }^{9,10}$ On the other hand, a large amount of previous research has revealed the serious consequences that sleep deprivation may have upon cognitive health. ${ }^{11,12}$ In general, sleep deprivation has been associated with impairments in working memory, alertness, declarative memory, higher-order cognitive skills, divergent thinking, and decision making. ${ }^{13-15}$ Indicatively, Drummond and Brown ${ }^{16}$ and Drummond et al ${ }^{17,18}$ examined the effects of sleep deprivation under two conditions: the participants in their studies were studied in a well-rested condition (six nights with 9 hours of time in bed each night) and one of two randomly assigned sleep deprivation conditions, namely, partial sleep deprivation (four nights with 4 hours of time in bed each night) or the "classical" total sleep deprivation condition (one night with no sleep). Their results suggest that dimensions of broader cognitive functions are differentially vulnerable to the effects of sleep deprivation, and different conditions of sleep deprivation could affect cognitive functions to different degrees. Beyond the differentiations, in general, they have shown that sleep deprivation could result in significant impairment in the activation of the prefrontal cortex and the structures that support working memory. A reduced temporal activation supporting verbal memory was also observed, together with an increased activation of areas of the parietal lobe. These results appear to support the existence of a certain neurophysiological mechanism of individual compensatory reactions utilizing posterior cortical systems, mainly in case of total sleep deprivation. ${ }^{18,19}$ The association of loss of sleep with affective functioning has attracted far less interest, although loss of sleep has been often associated with emotional irritability and behavioral variability. ${ }^{12,20}$ Killgore et $\mathrm{al}^{21}$ found significant decreases, after sleep deprivation in the form of 55+ hours of continuous wakefulness, in the emotional intelligence of the young adult participants in their study. Specifically, they found decreases in intrapersonal functioning (reduced sense of self-esteem and self-confidence), interpersonal functioning (decreased empathy toward others and low quality of interpersonal relations), stress management skills (impaired control of impulses), and behavioral treatment (reduced positive thinking and limited exposure to action), together with increased internal reflection (greater dependence on superstitions and magical thinking processes). In addition, Kahn-Greene et al, ${ }^{22}$ using an experimental design, found that sleep deprivation in the form of 55 hours of continuous wakefulness caused increased tendency to blame others and reduced tendency to normalize tensions in a fight by accepting responsibility for an action, compared to the results with rested baseline. The aforementioned data suggest that sleep deprivation weakens both inhibition of aggression and willingness to behave in ways that facilitate effective social interaction, probably due to reduced metabolic activity in prefrontal cortex, mainly in brain regions responsible for inhibitory behavior, affect, and personality.

Specifically, in the context of the ability to recognize emotions, Van der Helm et $\mathrm{al}^{23}$ asked the participants in their study to evaluate several visual stimuli by labeling the expressed emotion (happiness, sadness, or anger) and evaluating the intensity (from neutral to severe emotional) of the expression. In the experimental group deprived of sleep for 30 hours, the task was performed once under conditions of sleep deprivation and twice under sleep-rested conditions. In the control group, the task was performed twice under sleep-rested conditions. The results showed a statistically significant decrease in the recognition of emotions of anger and happiness under sleep deprivation, but only for the visual stimuli of moderate intensity. Nevertheless, any deficits that occurred were ameliorated following one night of sleep.

Based on these studies, it is obvious that the majority of the empirical work on the loss of sleep refers to young adults who are usually examined under "total sleep deprivation" and "at rest" conditions. However, it is common knowledge that, as humans age, their bodies go through radical changes. As one of the consequences, the more the age of the adult person increases, the fewer are the hours of night sleep they experience, and this is associated with deterioration of cognitive abilities. ${ }^{24}$ Hence, it seems that sleep is more important for older adults, compared to younger ones, in order to maintain their cognitive and affective functioning at an adequate level, to offset the aggravating effects of aging. 
However, considering that the hours of sleep are reduced in older adults, the supportive role of night sleep in cognition and affect could be restricted in terms of hours (or time) of the day, compared to the same in younger adults.

\section{The effects of the ToD condition on cognition and affect}

The majority of research on sleep examines the impact of loss of sleep on human body and functions, rather than the benefits of sleep on them. Only a limited number of studies - a few decades ago - have moved toward this direction by utilizing the variable ToD. In general, it seems that not everyone performs equally effectively at all hours of the day. Some people prefer the morning (morning types), whereas others prefer the afternoon (afternoon or evening types). This variation is primarily due to individual differences. Horne and Ostberg ${ }^{25}$ developed a self-report questionnaire to rank people in the dimension of "morning - evening types (morningness - eveningness)". They found that those classifying themselves as "evening types", started the day with a lower body temperature than that of the "morning types", touching the highest temperature at $8.30 \mathrm{pm} .{ }^{26}$ Using the same questionnaire, Petros et $\mathrm{al}^{27}$ found that recall of prose texts, which are considered highly demanding tasks for working memory, is reduced during the day for the morning types, while increasing for the afternoon types. ToD seems to also affect older adults' performance in tasks that require cognitive processing. Specifically, a series of studies has found significant effects of this variable on short-term memory, ${ }^{28}$ attention, ${ }^{29}$ inhibitory control, ${ }^{30}$ and semantic activation. ${ }^{31}$

Besides the strict aspects of cognition, the role of sleep in mixed cognitive-affective brain processes has only very recently become the focus of research interest. ${ }^{32}$ Regarding emotion recognition, in particular, Gujar et al, ${ }^{9}$ using a face recognition task, revealed an amplified reactivity of young adults to anger and fear across the day, without sleep. However, an intervening nap was found to block this reactivity to anger and fear, while enhancing ratings of happy expressions. Moreover, it was found that only those who attained REM sleep displayed this remodulation of affective reactivity for anger and fear. On the basis of these findings, Gujar et $\mathrm{al}^{9}$ suggest that emotion recognition is not static across a daytime waking interval, showing a progressive reactivity toward negative expressions. However, an episode of REM sleep can reverse this predisposition, supporting the claim that REM neurophysiology may represent a decisive factor for the optimal homeostasis of affective brain processes. ${ }^{33}$ However, again, the majority of the studies on the relationships between the
ToD condition and affective or/and mixed cognitive-affective brain processes refer to young adults.

With the advancing of age, a change in the preferred ToD is observed, with older adults performing tasks requiring cognitive processes at a higher level in the morning. ${ }^{34,35}$ Moreover, in another study, West et $\mathrm{al}^{36}$ found that older adult participants rated themselves as mainly morning types, while young adults rated themselves as neutral. In the same study, older adults displayed higher-level performance in cognitive tasks when those tasks were administered in the morning, compared to tasks administered in the afternoon, when the influence of disruptive stimuli was found to be greater. Beyond self-reports and performance in neuropsychological tests, the classification of older adults as morning types is well supported by physiological changes related to aging. Specifically, there is clear evidence regarding age-related changes in circadian rhythms, according to which the phase of the circadian rhythms of older adults becomes earlier (phase shift toward a morning chronotype) and their amplitude reduces. ${ }^{37-39}$ There is loss of rhythmicity in a series of hormones. For example, many studies have found a decline in melatonin production and a subsequent reduction in amplitude of the circadian melatonin rhythm in aging, which is considered indicative of a biological clock that is running down as the organism arrives at the end of its life. ${ }^{37-39}$ On the other hand, recent research on molecular rhythms in the aging brain has identified a set of genomewide transcripts that gain a circadian rhythm in expression in prefrontal cortex only in older adults. It has been claimed that this may represent a compensatory mechanism of the aging organism toward deregulation of its biological clock. ${ }^{39}$ However, besides compensation, the same research has found that $>1,000$ genes exhibit alterations in rhythmicity patterns with advancing age, and this may contribute to altered sleep, cognition, and affect in aging. ${ }^{39}$

Therefore, based on the aforementioned context, there is evidence regarding the associations among altered sleep, altered preferred ToD, and changes in cognitive performance and affective experience in aging, at both the neuropsychological and the physiological levels. Nevertheless, to our knowledge, there are no studies examining the effects of sleep and ToD on mixed cognitive-affective processes, such as emotion decoding from dynamic, ecologically valid visual cues, in older adults.

\section{The aim of the study}

Hence, considering the fewer hours of night sleep that older adults experience and the association of the reduced hours of sleep with deterioration of cognitive abilities, on the one 
hand, as well as their self-reported categorization as morning types and their higher-level performance in cognitive tasks in the morning, on the other hand, this study aimed at examining the combined effects of night sleep and ToD on emotion decoding from dynamic cues, in aging. Taking into account that emotion recognition in young adults seems not to be static across a daytime waking interval, the main hypothesis of the study was formulated as follows. Older adults would show a higher-level ability to decode basic emotions from dynamic visual cues in the morning and after night sleep, than in the afternoon and after many hours of being awake (Hypothesis 1).

\section{Methods}

\section{Design}

The study followed a within-subjects design, according to which the same participants were examined twice: first condition: "early in the morning and after night sleep", vs second condition: "in the afternoon and after many hours since night sleep". The participants were assigned to each experimental condition in that way so that half of them completed the assignment in the morning first, and the other half in the afternoon first. The two conditions were separated with a timeline of 1 week, in order to avoid any potential mnemonic biases. The participants abstained from caffeine and alcohol for 72 hours before and during the conduct of each condition of the study, while they preserved a normal sleep-wake rhythm, with the mean of the sleeping phase fluctuating between 5 hours and 7 hours. Any intervening nap was not permitted/allowed to take place before the examination in the afternoon.

\section{Participants}

Thirty-seven community-dwelling older adults, free of a diagnosis related to cognitive decline, participated in the study. It was a convenience sample recruited from an Open Care Center for Older Adults in Thessaloniki, Greece (age range: $60-80$ years, mean $=69.6$ years, $\mathrm{SD}=5.4$ years; educational level: $24.3 \%$ low (0-9 years of formal education), 59.5\% middle (10-12 years of formal education), and $16.2 \%$ high ( $\geq 13$ years of formal education); sex: 19 women and $18 \mathrm{men})$.

First, the Montreal Cognitive Assessment in Greek (MoCA $)^{40}$ and the Greek version of the Geriatric Depression Scale-15 (GDS-15) ${ }^{41,42}$ were administered to possible participants, to screen for persons with probable mild cognitive impairment and depressive symptomatology, respectively, and exclude them from the final sample. Persons with a MoCA score $\geq 26$ (range: $26-30$, mean $=27.6, \mathrm{SD}=1.4$ ) or/and a GDS-15 score $\leq 6$ (range: $0-6$, mean $=2.3, \mathrm{SD}=1.4$ ) were included in the final sample and were subsequently examined twice via the main tool of the study. Additional criteria for exclusion were a self-reported history of neurological or psychiatric disorder, any self-reported history of sleep disorder or drug abuse, any current intake of sleeping pills or antidepressants, severe head trauma, profound visual impairments, and verbal incomprehension.

\section{Ethical standards}

The authors assert that all procedures contributing to this work comply with the ethical standards of the relevant national and institutional committees on human experimentation and with the Helsinki Declaration of 1975, as revised in 2008. All the participants were informed about the procedure and the aim of the study, and their written informed consent was taken.

\section{Procedure}

The two conditions of the experiment were conducted in a place of the participant's choice. In every case, a quiet room was selected, to avoid distractions. The exact ToD for the two examinations was decided after personal communication with every participant. In detail, with regard to the first condition, namely, the condition "early in the morning and after night sleep", the participants performed an emotion recognition test (described later) at 8 am ( \pm 30 minutes), on average, 2 hours after waking up. As regards the second condition, ie, the condition "in the afternoon and after many hours since night sleep", the participants were examined using the same test at $5.30 \mathrm{pm}$ ( \pm 30 minutes), on average, 11.5 hours after waking up. In both the conditions, every participant completed the task individually.

\section{Instrument}

The Emotion Evaluation Task (EET, Form A) ${ }^{5,43-45}$ was used to examine the ability to decode basic emotions from ecologically valid cues. The TASIT Part I: EET (Form A) is the first part of a broader audiovisual tool developed for the clinical assessment of social perception, called the The Awareness of Social Inference Test (TASIT).

The EET-Form A (or, simply, the EET) comprises 28 short (15-60 seconds) videotaped vignettes of people interacting in everyday situations. In fact, the people are "method actors" who have been trained to elicit real emotions in themselves. It is worth mentioning that actors were specifically trained not to provide the viewer with unlimited exposure to an emotional expression, because, in real life, displays of genuine emotion are fleeting and evolving and usually mediated by a tendency to "play against" expressions of strong emotion. Hence, all the emotions expressed by the actors are 
of moderate intensity. The EET also uses visual and auditory contextual cues to assess emotion decoding systematically. In some scenes, there is only one actor talking. Other scenes depict two actors. In these cases, instructions are given to focus on one actor. ${ }^{5,43-45}$

The "target" actor in each scene enacts the script according to one of six emotions (happiness, pleasant surprise, sadness, anger, anxiety, and disgust/revulsion) or no particular emotion (ie, neutral). After viewing each scene, the participant is asked to choose the emotion displayed by the actor from among a list of six emotional categories and one nonemotional category (neutral) displayed on a response card. There are five response cards, and each card presents the seven categories in a different order. The 28 scenes comprise four portrayals of each emotion, as well as the neutral condition, in quasirandomized order. All the scripts are neutral in content and do not lend themselves to any specific emotion. However, as the test was developed in English, we decided to administer it with the sound turned off, so as to focus on the participant's ability to recognize only dynamic visual cues. Administration of the EET typically takes 15-25 minutes, depending on the participant's age and condition. The EET was administered according to the standard procedure outlined in the relevant manual. To familiarize the participant with the task requirements, a practice item preceded the main task. ${ }^{5}$ The test-retest reliability of the EET's psychometric qualities, based on the performance of adults with severe traumatic brain injury, was estimated to be moderate ( $r=0.74)$. In relation to the EET's construct validity, it was found to be significantly correlated with premorbid intelligence quotient (IQ), information processing speed, executive functions, new learning of social information (specifically, faces), and the Ekman labeling and matching of photos as measures of social perception. ${ }^{43-45}$ As regards the structural validity of the EET when it is administered as a test for decoding only visual cues, Moraitou et $a l,{ }^{5}$ using structural equation modeling, confirmed a model for the factorial structure of the EET in adults. According to this model, it seems that decoding of all basic emotions - except happiness - from dynamic visual cues follows the same neuropsychological mechanisms, and a basic step in emotion decoding involves recognizing whether information presented is emotional or not. In the same study, age was found to negatively affect the ability to decode from dynamic visual cues all the basic emotions except happiness. ${ }^{5,46-48}$

\section{Results}

The data gathered after EET administration to the sample were first analyzed by applying a $2 \times 7$ repeated-measures analysis of variance (ANOVA), with the within-subjects variables of experimental condition (two levels: "early in the morning and after night sleep" vs "in the afternoon and after many hours since night sleep") and emotion type (seven levels: happy, surprised, angry, anxious, sad, disgusted, and neutral) as the independent variables, and emotion decoding performance as the dependent variable. Mauchly's test indicated that the assumption of sphericity had not been violated either for the main effect of emotion type or for the interaction between condition type and emotion type. The analysis indicated that there was a significant main effect of condition type on emotion decoding: $F(1,36)=386.92$, $P<0.001, \eta^{2}=0.92$; as well as of emotion type on emotion decoding: $F(6,31)=24.32, P<0.001, \eta^{2}=0.83$. There was also a significant interaction effect between condition type and emotion type: $F(6,31)=25.94, P<0.001, \eta^{2}=0.83$. This indicates that condition type had different effects on emotion decoding from dynamic visual cues depending on which specific emotion was asked to be recognized.

To break down the interaction and shed light on the specific differences due to condition type or emotion type, a series of one-way repeated-measures ANOVAs were conducted. First, seven repeated-measures ANOVAs with the within-subjects variable of experimental condition (two levels: "early in the morning and after night sleep" vs "in the afternoon and after many hours since night sleep") as the independent variable, in addition to each one of the six emotion types and the emotionally neutral condition as the dependent variable, were conducted. The results showed that emotion decoding from dynamic visual cues, in terms of all the emotions and the neutral condition, was significantly affected by the experimental condition: for happiness: $F(1,36)=4.36, P<0.05, \eta^{2}=0.11$; for pleasant surprise: $F(1,36)=166.24, P<0.001, \eta^{2}=0.82$; for neutral expression: $F(1,36)=31.57, P<0.001, \eta^{2}=0.47$; for sadness : $F(1,36)=170.95, P<0.001, \eta^{2}=0.83$; for anger: $F(1,36)=37.10$, $P<0.001, \eta^{2}=0.51$; for anxiety: $F(1,36)=40.61, P<0.001$, $\eta^{2}=0.53$; and for disgust: $F(1,36)=14.58, P=0.001$, $\eta^{2}=0.29$. Relative to their performance in emotion recognition early in the morning and after night sleep, older adult participants had a significantly lower ability of correct recognition of all basic emotions - including happiness - as well as of the emotionally neutral condition, in the afternoon and after many hours of remaining awake (Figure 1).

In the next step, the data gathered after EET administration in the first condition (in the morning and after night sleep) were analyzed by applying a repeated-measures ANOVA, in which the within-subjects factor of emotion type was the independent variable and emotion-decoding performance 


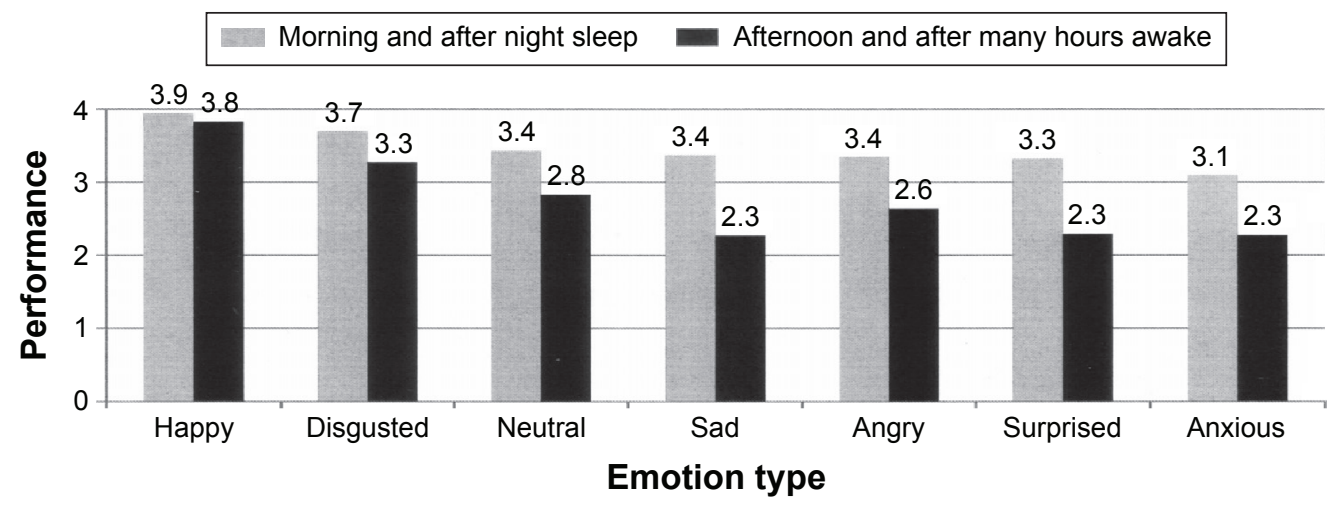

Figure I Older adults' emotion recognition ability in two conditions in terms of sleep and time of day.

was the dependent variable. The same type of analysis was conducted for the data gathered by EET administration in the second condition (in the afternoon and after many hours of being awake). These analyses revealed that there was a significant effect of emotion type on emotiondecoding performance in both experimental conditions. For Condition 1 (in the morning and after night sleep), Mauchly's test indicated that the assumption of sphericity had been violated for the main effect of emotion type: $\chi^{2}(20)=44.70$, $P=0.001, \varepsilon=0.83$; therefore, multivariate tests are reported: $V=0.67, F(6,31)=10.39, P<0.001, \eta^{2}=0.67$; for Condition 2 (in the afternoon and after many hours of being awake): $F(6,31)=38.71, P<0.001, \eta^{2}=0.88$. In terms of the pattern of the emotion type effects, for Condition 1 (Figure 1), pairwise comparisons with Bonferroni correction showed that older adults' greater performance in the morning was in the recognition of happiness, followed by recognition of revulsion/ disgust (Figure 1). Recognition of happiness was found to differ significantly from recognition of all other emotions including the neutral condition $(P<0.001-0.003)$ - except disgust, the recognition of which was found to be significantly higher from anxiety recognition $(P<0.05)$.

With regard to the pattern of the emotion type effects, in Condition 2 (Figure 1), pairwise comparisons with Bonferroni correction showed that it was relatively similar to the pattern revealed in Condition 1. Specifically, older adults' greater performance in the afternoon was displayed again in happiness recognition, followed by revulsion/disgust (Figure 1). Happiness recognition was found to be significantly higher than recognition of all other emotions and the neutral condition $(P<0.001)$ - including disgust $(P<0.01)$. Disgust recognition was also found to be significantly higher, compared with recognition of the remaining four emotions $(P<0.001-0.002)$, but not in comparison with recognition of the emotionally neutral condition. Neutrality discrimination was significantly higher, compared with sadness recognition $(P<0.03)$. Hence, the most important findings of this study seem to be the quantitative differences between the recognition of the same type of emotion in the two experimental conditions, rather than any preferences to decode specific emotions or emotions of specific valence, as a result of probable sleep and ToD influences.

\section{Discussion}

This study aimed at examining the combined effects of night sleep and ToD variables on emotion recognition ability in older adults. Emotion recognition is a relatively primitive ability, ${ }^{1,2}$ which, however, is very important for supporting complex social cognition skills and empathy. For this purpose, a within-subjects field experiment was designed in which the same older adult participants were examined twice, ie, "in the morning and after night sleep" and "in the afternoon and after many hours of being awake", in terms of emotion recognition. The main advantage of the study was the use of a dynamic, ecologically valid instrument to measure the ability to discriminate between nonemotional expressions and decode basic emotions from visual cues that are very similar to those encountered in everyday life. The results clearly indicate that older adults possess a significantly higher-level ability to decode basic emotions from dynamic visual cues in the morning and after night sleep, than in the afternoon and after many hours of being awake, thus totally confirming Hypothesis 1.

The finding that there is a generalized decline in emotion recognition ability is an indication that a broader network of brain areas and processes supporting emotion recognition are negatively affected by the condition "afternoon and many hours of being awake". It is noteworthy that even happiness recognition is reduced, considering that decoding of happiness from dynamic visual cues was found to be excellently 
resilient to the aging process ${ }^{5}$ while being substantially decreased only in neurodegenerative conditions such as dementia and stroke. ${ }^{47,48}$ Such findings corroborate previous research suggesting that older adults are morning types in terms of their higher-level performance in tasks requiring a series of cognitive processes. ${ }^{28-30}$ Moreover, the gap/interval of several hours from night sleep, without an intervening nap, may function as sleep deprivation condition for older adults, resulting in all the disadvantages for cognition that have been described by a large number of studies. ${ }^{12,13}$

More specifically, as already mentioned, the EET is designed to examine emotion decoding from dynamic displays of genuine emotions that are fleeting and evolving. ${ }^{43-45}$ Considering that according to studies that investigated the neural regions involved when people decode emotions that change dynamically, frontal lobe regions, such as the orbitofrontal cortex and the gyrus rectus, are primarily involved in such a process ${ }^{49}$ it seems that executive functions are mostly affected by the condition "afternoon and many hours of being awake", because these cognitive processes are mainly supported by frontal networks. This conclusion is along the same lines as the suggestion that the main type of impairment in the sleep deprivation condition is in the activation of the prefrontal regions that support working memory and inhibitory control. ${ }^{16-18,22}$

In addition to the general picture, Figure 1 shows clearly that the most difficult-to-be-recognized emotions in the condition "afternoon and many hours of being awake" were sadness, anxiety, and pleasant surprise. This finding seems not in line with previous research on the effects of sleep deprivation and ToD on specific emotion decoding in young adults, which revealed divergent influences of $\mathrm{ToD}$ and sleep deprivation only on some of the basic emotions and not on the same with these found in the present study. ${ }^{9,23}$ A partial explanation of this inconsistency could be given at the methodological level, in terms of the measures used and the age of the samples of these studies. In addition to the methodological issues, it seems that the three emotions of interest have in common a relatively high level of uncertainty: considering the moderate intensity of all emotions displayed in the EET ${ }^{5}$ and previous findings regarding older adults' tendencies to confuse emotionally neutral cues with anxiety, pleasant surprise, and sadness, ${ }^{47,48}$ it is obvious that the three emotions - at least as presented in the EET - are ambiguous enough. Hence, it could be supported that $\mathrm{ToD}$ and sleep variables may influence especially the recognition of emotional cues characterized by relatively high unexpectedness. ${ }^{5,50,51}$ At a deeper level, this might mean that brain regions involved in uncertainty processing, such as the fusiform gyrus, ${ }^{50-53}$ decrease their activation under the condition "afternoon and many hours of being awake", at least in older adults.

Moreover, on the same wavelength with the findings of this study, behavioral and neurophysiological data in a recent empirical work on the effects of sleep deprivation on recognition of happiness, anger, fear, and sadness in young adults showed that sleep deprivation had a preferentially negative impact on sadness decoding. A possible explanation for such a finding, which could be generalized to anxiety as well, is that decoding difficulties may be associated with the relatively low levels of physiological arousal that the specific emotions elicit. ${ }^{9,54}$ On the basis of the extant literature, it seems that following sleep loss, broader brain areas are engaged in order to accurately identify high-arousal emotional cues. Nevertheless, such compensation is not achieved for emotional cues eliciting lower arousal, perhaps because they are not experienced by an organism as crucial for survival and therefore, deserving to invest cognitive resources, when these are limited by a challenge such as sleep loss. ${ }^{9,54}$ In addition to neurocognitive and evolutionary perspectives, according to socioemotional approaches, sadness, anxiety, and surprise can be considered as highly self-relevant emotions that are characterized by the "unpleasant" dimensions of negative valence or/and high unpredictability. Due to motivational shifts toward affect regulation and well-being, older adults do not prefer to experience and, therefore, process such emotions in any way, because they consider they are threatening to the regulation of their affect and maintenance of their well-being. ${ }^{54-56}$ This "choice" may become more automated when their already limited cognitive resources become even more limited. ${ }^{55,56}$

On the other hand, considering the qualitatively similar patterns of effects of emotion type on emotion recognition found for both experimental conditions (Figure 1), the notion that the changes in emotion recognition ability across a daytime interval reflect a progressive reactivity toward negative expressions ${ }^{9}$ is not supported. In fact, the results show that recognition of happiness and disgust is maintained at a high level in both conditions, compared to the recognition of all the other emotions, including the emotionally neutral expressions and behavior. This finding could be explained by the different and probably more resistant-to-aging brain networks that might be involved in, and support, happiness and disgust decoding, as well as by socioenvironmental factors. ${ }^{?}$ In addition to this, the results of this study clearly confirm the suggestion that emotion recognition ability is subject to changes across a daytime waking interval. ${ }^{9}$ 


\section{Limitations and future research}

The preliminary nature of this study is the major limitation. The study was based on a small, convenience sample of participants and on a field-type experiment, and therefore, the findings are essentially tentative. Replication of these findings in a more representative sample of older adults in a laboratory experiment, by using objective measures of sleep and neuroimaging methods, together with neuropsychological testing, is needed to increase confidence in these findings. Furthermore, more complex experimental designs, including varying sleep conditions, should be developed to find ways that could help older adults to maintain a high level of emotion decoding ability during the whole day.

\section{Conclusion}

The results of the present study clearly support the claim that emotion recognition ability is dynamic and subject to changes across a daytime waking interval. More specifically, the study contributes to the extant literature by indicating that older adults possess a significantly higher-level ability to decode basic emotions from dynamic visual cues in the morning and after night sleep, compared to that in the afternoon and after many hours of being awake. In addition to theoretical interest, this finding is important for the everyday life of older adults. It becomes obvious that due to emotion recognition deficits, older adults may not achieve successful social interactions when they are awake for many hours and have to behave as evening types. In this vein, appropriate interventions could be developed and directions should be given (eg, for the time and the duration of an intervening nap) to ameliorate the negative effects of sleep deprivation and $\mathrm{ToD}$ variables.

\section{Disclosure}

The authors report no conflicts of interest in this work.

\section{References}

1. Adolphs R, Baron-Cohen S, Tranel D. Impaired recognition of social emotions following Amygdala damage. J Cogn Neurosci. 2002;14(8): 1264-1274.

2. Mill A, Allik J, Realo A, Valk R. Age-related differences in emotion recognition ability: a cross-sectional study. Emotion. 2009;9(5): 619-630.

3. Adolphs R. How do we know the minds of others? Domain specificity, simulation, and enactive social cognition. Brain Res. 2006; 1079(1):25-35.

4. Ruffman T, Henry JD, Livingstone V, Phillips LH. A meta-analytic review of emotion recognition and aging: implications of neuropshycological models of aging. Neurosci Biobehav Rev. 2008;32(4):863-881.

5. Moraitou D, Papantoniou G, Gkinopoulos T, Nigritinou M. Older adults' decoding of emotion: age-related differences in interpreting dynamic emotional displays and the well preserved ability to recognize happiness. Psychogeriatrics. 2013;13(3):139-147.
6. Ko SG, Lee TH, Yoon HY, Known J-H, Mather M. How does context affect assessment of facial emotion? The role of culture and age. Psychol Aging. 2011;26(1):48-59.

7. Suzuki A, Hoshiro T, Shigemasu K, Kawamura M. Decline or improvement? Age-related differences in facial expression recognition. Biol Psychol. 2007;74(1):75-84.

8. Halberstadt J, Ruffman T, Murray J, Taumoepeau M, Ryan M. Emotion perception explains age-related differences in the perception of social gaffes. Psychol Aging. 2011;26(1):133-136.

9. Gujar N, McDonald SA, Nishida M, Walker MP. A role for REM sleep in recalibrating the sensitivity of the human brain to specific emotions. Cereb Cortex. 2011;21(1):115-123.

10. $\mathrm{Hu}$ P, Stylos-Allan M, Walker MP. Sleep facilitates consolidation of emotional declarative memory. Psychol Sci. 2006;17(10):891-898.

11. Durmer JS, Dinges DF. Neurocognitive consequences of sleep deprivation. Semin Neurol. 2005;25(1):117-129.

12. Zohar D, Tzischinsky O, Epstein R, Lavie P. The effects of sleep loss on medical residents' emotional reactions to work events: a cognitiveenergy model. Sleep. 2005;28(1):47-54.

13. Dinges DF. Probing the limits of functional capability: the effects of sleep loss on short-duration tasks. In: Broughton RJ, editor. Sleep, Arousal, and Performance. Boston: Birkha; 1992:177-188.

14. Harrison Y, Horne JA. The impact of sleep deprivation on decision making: a review. J Exp Psychol Appl. 2000;6(3):236-249.

15. Van Dongen HP, Maislin G, Mullington JM, Dinges DF. The cumulative cost of additional wakefulness: dose-response effects on neurobehavioral functions and sleep physiology from chronic sleep restriction and total sleep deprivation. Sleep. 2003;26(2):117-126.

16. Drummond SP, Brown GG. The effects of total sleep deprivation on cerebral responses to cognitive performance. Neuropsychopharmacology. 2001;25(5):68-73.

17. Drummond SP, Brown GG, Gillin JC, Stricker JL, Wong EC, Buxton RB. Altered brain response to verbal learning following sleep deprivation. Nature. 2000;40(3):655-657.

18. Drummond SP, Brown GG, Stricker JL, Buxton RB, Wong EC, Gillin JC. Sleep deprivation-induced reduction in cortical functional response to serial subtraction. Neuroreport. 1999;10(18):3745-3748.

19. Drummond SP, Gillin JC, Brown GG. Increased cerebral response during a divided attention task following sleep deprivation. J Sleep Res. 2001;10(2):85-92.

20. Baldwin DC Jr, Daugherty SR. Sleep deprivation and fatigue in residency training: results of a national survey of first- and second-year residents. Sleep. 2004;27(2):217-223.

21. Killgore WD, Kahn-Greene ET, Lipizzi EL, Newman RA, Kamimori GH, Balkin TJ. Sleep deprivation reduces perceived emotional intelligence and constructive thinking skills. Sleep Med. 2008; 9(5):517-526.

22. Kahn-Greene ET, Lipizzi EL, Conrad AK, Kamimori GH, Killgore WD. Sleep deprivation adversely affects interpersonal responses to frustration. Pers Indiv Differ. 2006;41:1433-1443.

23. Van der Helm E, Gujar N, Walker MP. Sleep deprivation impairs the accurate recognition of human emotions. Sleep. 2010;33(3): 335-342.

24. Ohayon MM, Vecchierini MF. Normative sleep data, cognitive function and daily living activities in older adults in the community. Sleep. 2005;28(8):981-989.

25. Horne JA, Ostberg O. Self-assessment questionnaire to determine morningness-eveningness in human circadian rhythms. Int J Chronobiol. 1976;4(2):97-110.

26. Horne JA, Ostberg O. Individual differences in human circadian rhythms. Biol Psychol. 1977;5(3):179-190.

27. Petros T, Beckwith W, Anderson M. Individual differences in the effects of time of day and passage difficulty on prose memory in adults. Br J Psychol. 1990;81:63-72.

28. Baddeley AD, Hatter JE, Scott D, Snashall A. Memory and time of day. Q J Exp Psychol. 1970;22:605-609.

29. Blake MJF. Time of day effects on performance in a range of tasks. Psychon Sci. 1967;9:349-350. 
30. May CP, Hasher L. Synchrony effects in inhibitory control over thought and action. J Exp Psychol Hum Percept Perform. 1998;24(2): 363-379.

31. Anderson MJ, Petros TV, Beckwith BE, Mitchell WW, Fritz S. Individual differences in the effect of time of day on longterm memory access. Am J Psychol. 1991;104:241-255.

32. Wiesner CD, Pulst J, Krause F, et al. The effect of selective REM-sleep deprivation on the consolidation and affective evaluation of emotional memories. Neurobiol Learn Mem. 2015;122:131-141.

33. Groch S, Wilhelm I, Diekelmann S, Born J. The role of REM sleep in the processing of emotional memories: evidence from behavior and event-related potentials. Neurobiol Learn Mem. 2013;99:1-9.

34. May CP, Hasher L, Stoltzfus ER. Optimal time of day and the magnitude of age differences in memory. Psychol Sci. 1993;4:326-330.

35. Mecacci L, Zani A, Rocchetti G, Lucioli R. The relationship between morningness-eveningness, aging, and personality. Pers Individ Dif. 1986;7:911-913.

36. West R, Murphy KJ, Armilio ML, Craik FIM, Stuss DT. Effects of time of day on age differences in working memory. J Gerontol B Psychol Sci Soc Sci. 2002;57B(1):3-10.

37. Fonseca Costa S, Ripperger JA. Impact of the circadian clock on the aging process. Front Neurol. 2015;6(43):1-5.

38. Monk TH. Aging human circadian rhythms: conventional wisdom may not always be right. J Biol Rhythms. 2005;20(4):366-374.

39. Chen CY, Logan RW, Ma T, et al. Effects of aging on circadian patterns of gene expression in the human prefrontal cortex. Proc Natl Acad Sci US A. 2016;113(1):206-211.

40. Nasreddine ZS, Chertkow H, Phillips N, Whitehead V, Collin I, Cummings JL. The Montreal cognitive assessment (MoCA): a brief cognitive screening tool for detection of mild cognitive impairment. Neurology. 2004;62(7):A132.

41. Fountoulakis KN, Tsolaki M, Iacovides A, et al. The validation of the short form of the Geriatric Depression Scale (GDS) in Greece. Aging (Milano). 1999;11(6):367-372.

42. Yesavage JA, Brink TL, Rose TL, et al. Development and validation of a geriatric depression screening scale: a preliminary report. JPsychiatr Res. 1982-1983;17(1):37-49.

43. McDonald S, Bornhofen C, Shum D, Long E, Saunders C, Neulinger K Reliability and validity of The Awareness of Social Inference Test (TASIT): a clinical test of social perception. Disabil Rehabil. 2006; 28(24):1529-1542.
44. McDonald S, Flanagan S, Rollins J, Kinch J. TASIT: a new clinical tool for assessing social perception after traumatic brain injury. J Head Trauma Rehabil. 2003;18(3):219-238.

45. McDonald S, Flanagan S, Rollins J; the University of New South Wales' Media \& Education Technology Support Unit. The Awareness of Social Inference Test (TASIT): Manual. London: Pearson; 2002.

46. Banzigrer T, Grandjean D, Scherer K. Emotion recognition from expressions in face, voice and body: the Multimodal Emotion Recognition Test (MERT). Emotion. 2009;9(5):691-704.

47. Gkinopoulos T, Moraitou D, Papantoniou G, Nigritinou M, Ginos P, Kotselidou D. Decoding of basic emotions from dynamic visual displays in dementia: a sign of loss of positivity bias in emotional processing in cognitively unhealthy aging? Open J Med Psychol. 2014;3:325-336.

48. Sismanidi A, Konstantinidou G, Miskedaki A, et al. Emotion decoding from dynamic visual cues in older adult stroke patients: a preliminary study. Hell J Nucl Med. 2015;18:250-257.

49. Goodkind MS, Sollberger M, Gyurak A, et al. Tracking emotional valence: the role of the orbitofrontal cortex. Hum Brain Mapp. 2012; 33(4):753-762.

50. Kim H, Somerville LH, Johnstone T, Alexander A, Whalen PJ. Inverse amygdala and medial prefrontal cortex responses to surprise faces. Neuroreport. 2003;14(18):2317-2322.

51. Kim H, Somerville LH, Johnstone T, et al. Contextual modulation of amygdala responsivity to surprised faces. J Cogn Neurosci. 2004; 16(10):1730-1745.

52. Ruffman T. Ecological validity and age-related change in emotion recognition. J Nonverbal Behav. 2011;35:297-304.

53. Wright C, Negreira A, Gold A, Britton J, Williams D, Feldman Barrett L. Neural correlates of novelty and face-age effects in young and elderly adults. Neuroimage. 2008;42(2):956-968.

54. Cote KA, Mondloch CJ, Segreeva V, Taylor M, Semplonius T. Impact of total sleep deprivation on behavioural neural processing of emotionally expressive faces. Exp Brain Res. 2014;232(5):1429-1442.

55. Moraitou D, Papantoniou G. Mindfulness and action control in young, middle-aged, and old adults: an examination of their relationship to attentional bias towards emotional information. Res Psychol. 2010;4: 591-626.

56. Moraitou D, Efklides A. Affect and emotions. In Fernandez-Ballesteros R, editor. GeroPsychology: European perspectives for an ageing world. Goettingen, Germany: Hogrefe \& Huber; 2007:82-102.
Neuropsychiatric Disease and Treatment

\section{Publish your work in this journal}

Neuropsychiatric Disease and Treatment is an international, peerreviewed journal of clinical therapeutics and pharmacology focusing on concise rapid reporting of clinical or pre-clinical studies on a range of neuropsychiatric and neurological disorders. This journal is indexed on PubMed Central, the 'PsycINFO' database and CAS,

\section{Dovepress}

and is the official journal of The International Neuropsychiatric Association (INA). The manuscript management system is completely online and includes a very quick and fair peer-review system, which is all easy to use. Visit http://www.dovepress.com/testimonials.php to read real quotes from published authors. 